\title{
Setting up a multidisciplinary program for management of cryptogenic stroke associated with patent foramen ovale
}

\author{
Mario Sičaja* \\ University Hospital Dubrava,
} Zagreb, Croatia
KEYWORDS: persistent foramen ovale, management, percutaneous closure. CITATION: Cardiol Croat. 2018;13(1-2):61. I https://doi.org/10.15836/ccar2018.61

*ADDRESS FOR CORRESPONDENCE: Mario Sičaja, Klinička bolnica Dubrava, Avenija Gojka Šuška 6, HR-10000 Zagreb, Croatia. / Phone: +385-91-406-0989 / E-mail: mario.sicaja@gmail.com

ORCID: Mario Sičaja, https://orcid.org/0000-0003-0773-4720

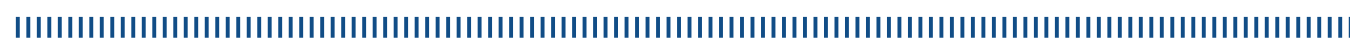

In the light of recent publications regarding patent foramen ovale (PFO) closure in patients with cryptogenic stroke, specifically regarding the results of trials CLOSE, REDUCE and RESPECT, there is an increasing need for optimal treatment of these patients., ${ }^{1,2}$ The aim of this presentation is to propose a multidisciplinary program regarding the diagnosis, treatment, and follow-up of patients with PFOmediated stroke based on a Heart-Brain team decision making. It is our aim to analyze necessary steps in everyday clinical practice, describe procedure work-flow together with tips-and-tricks regarding the procedure, and to review the activities during the first year of implementation in our practice. In conclusion, the first year of implementation of the above described program allowed us a quick implementation of published trial results in every day clinical practice together with high level of confidence, a short learning curve for a dedicated interventional cardiologist and with a superior patient safety. Furthermore, it allowed us to indicate the potential flaws in the protocol and practice, especially regarding reimbursement for healthcare insurance.
RECEIVED:

January 28, 2018

ACCEPTED:

February 10, 2018

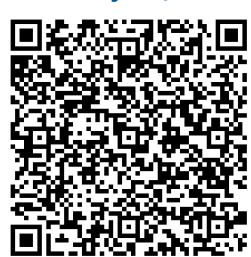

LITERATURE IIIIIIIIIIIIIIIIIIIIIIIIIIIIIIIIIIIIIIIIIIIIIIIIIIIIIIIIIIIIIIIIIIIIIIIIIIIIIIIIIIIIIIIIIIIIIIIIIIIII

1. Rigatelli G, Braggion G, Chinaglia M, Cardaioli P, Roncon L, Giordan M, et al. Setting up a multidisciplinary program for management of patent foramen ovale-mediated syndromes. J Interv Cardiol. 2006 Jun;19(3):264-8. https://doi.org/10.1111/j.1540-8183.2006.00141.x

2. Abo-Salem E, Chaitman B, Helmy T, Boakye EA, Alkhawam H, Lim M. Patent foramen ovale closure versus medical therapy in cases with cryptogenic stroke, meta-analysis of randomized controlled trials. J Neurol. 2018 Jan 22. [Epub ahead of print]. https://doi.org/10.1007/s00415-018-8750-x 